\title{
Time-Frequency Relationship
}

\section{between Inflation and Inflation Uncertainty for the U.S.: \\ Evidence from Historical Data}

\author{
Claudiu Tiberiu Albulescu ${ }^{\mathrm{a}}$, Aviral Kumar Tiwari ${ }^{\mathrm{b}}$, Stephen M. Miller ${ }^{\mathrm{c} 1}$ and Rangan Gupta ${ }^{\mathrm{d}}$ \\ ${ }^{a}$ Management Department, Politehnica University of Timisoara, P-ta. Victoriei No. 2, 300006, Timisoara, \\ Romania. \\ ${ }^{b}$ Faculty of Management, IBS Hyderabad, IFHE University, Dontanpalli, Hyderabad, Pin-501203, India. \\ ${ }^{c}$ Department of Economics, University of Nevada, Las Vegas, Las Vegas, Nevada, 89154-6005, USA. \\ ${ }^{d}$ Department of Economics, University of Pretoria, Pretoria, 0002, South Africa.
}

\begin{abstract}
We provide new evidence on the relationship between inflation and its uncertainty in the U.S. on an historical basis, covering the period 1775-2014. First, we use a bounded approach for measuring inflation uncertainty, as proposed by Chan et al. (2013), and we compare the results with the Stock and Watson (2007) method. Second, we employ the wavelet methodology to analyze the co-movements and causal effects between the two series. Our results provide evidence of a relationship between inflation and its uncertainty that varies across time and frequency. First, we show that in the medium- and long-runs, the FreidmanBall hypothesis holds when the measure of uncertainty is unbounded, while if the opposite applies, the Cukierman-Meltzer reasoning prevails. Second, we discover mixed evidence about the inflation-uncertainty nexus in the short-run, findings which explain the mixed results reported to date in the empirical literature.
\end{abstract}

Keywords: historical inflation rate, uncertainty, continuous wavelet transform, bounded series, U.S.

JEL code: C22, E31, N11, N12.

\footnotetext{
${ }^{1}$ Corresponding author. E-mail address: stephen.miller@unlv.edu.
} 


\section{Introduction}

During the 1960s, macroeconomists embraced the Phillips curve as a menu of outcomes that policy makers could select through the appropriate implementation of macroeconomic policy levers. That is, the Phillips curve at that time showed a trade-off between unemployment and inflation. In the 1970s, actual policy choices led to stagflation, which shattered the belief in the Phillips curve menu idea and led to the important role of inflation expectations and "inflation uncertainty". Friedman (1968) and Phelps (1967) independently predicted this outcome whereby the government's lack of commitment toward inflation influences price expectations, which shifts the short-run Phillips curve. Consequently, the variability of inflation over time depends on the limits established for an acceptable inflation rate.

The link between the level of inflation and its uncertainty became one of the focal points of economic literature, with the Nobel lecture of Friedman (1976) making two arguments about the inflation-unemployment trade-off. First, Friedman argues that no stable trade-off exists between inflation and unemployment. Second, he argues that higher inflation rates mean more inflation volatility and uncertainty (first hypothesis), which, in turn, reduces economic efficiency because of confused signals about the price changes (second hypothesis). More precisely, increasing inflation to achieve full employment creates a strong incentive to counter it, and generates an increased uncertainty about future inflation, as policies go from one direction to the other. Further, a higher uncertainty about inflation negatively affects output due to breakdowns in the price mechanism. Consequently, "this uncertainty - or more precisely, the circumstances producing this uncertainty - leads to systematic departures from the conditions required for a vertical Phillips curve" (Friedman, 1977, pp. 465).

Ball (1992) formalized the positive relationship between inflation and inflation uncertainty that Friedman (1977) highlighted, which now is known as the Friedman-Ball hypothesis. Ball (1992) argues that high inflation creates uncertainty about future monetary policy. In his asymmetric information model, he considers two types of policy makers, which fight against inflation (weak and strong types) and the public does not know the type of policymaker. Therefore, uncertainty exists regarding the actions the policy makers will take when faced with high inflation, which leads to increased inflation uncertainty.

Starting from Friedman's (1977) theory, Cukierman and Meltzer (1986) formulate an alternative hypothesis, showing that high inflation uncertainty leads to high inflation. They argue that in the presence of high inflation uncertainty, the monetary authority may create inflation surprises to stimulate real activity. Hence, even if the monetary authority has no 
incentive to create inflation, its attempts to stimulate the economy increase uncertainty about money and inflation, raising the level of inflation. Consequently, inflation uncertainty positively influences inflation.

Other competing theories propose a negative relationship between inflation and inflation uncertainty, contradicting the Friedman-Ball hypothesis. First, Frohman et al. (1981) point out that, if economic agents form their expectations on predictable variables other than past inflation, then high inflation variability accompanies low uncertainty. Second, Pourgerami and Maskus (1987) suggest that a rising inflation reduces inflation uncertainty because economic agents devote more resources to generate accurate predictions during inflationary periods. Ungar and Zilberfarb (1993) formalize these assumptions theoretically and show that high inflation does not necessarily determine high inflation uncertainty.

Finally, staring from the Friedman-Ball and Cukierman-Meltzer hypotheses, Holland (1995) demonstrates that data timing influences the link between inflation and its uncertainty. Relying on U.S. statistics, he shows that high inflation precedes greater uncertainty in some periods. In this situation, however, the monetary authority contracts the money supply growth rate to control welfare loses, which causes inflation to fall.

Though they differ in the direction of causality, the above mentioned hypotheses were all validated by empirical studies. The mixed results documented by the empirical literature reflect differences in methodologies (linear or non-linear models), in the measures of inflation uncertainty (see Cukierman and Meltzer, 1986), or in the time horizon for the inflation expectations (see Pourgerami and Maskus, 1987).

Against this background and to shed further light on the relationship between inflation and its uncertainty, we make four contributions to the existing literature. First, given the validation of different competing hypothesis by previous studies, we posit a non-linear link between the inflation rate and its uncertainty. Indeed, Kim (1993) shows that regime switching may provide the key element in explaining inflation uncertainty. Hypothesizing a high and low inflationary regime, however, is too simplistic and arbitrary. Moreover, a strict focus on the time-domain ignores the importance of the frequency-varying properties of inflation and its uncertainty. In addition, common models assume stationary time-series, which is not always the case. Therefore, to address these limitations, we propose a new analysis based on wavelets, which combines frequency and time domains. As far as we know, Bouoiyour and Selmi (2014) wrote the only paper that investigates the inflation-uncertainty nexus using the Maximal Overlap Discrete Wavelet Transform (MODWT) and a series of non-linear causality tests for Egypt. Our study differs in that we employ the Continuous 
Wavelet Transform (CWT) and its developments, which permits the identification of nonlinearities and structural breaks in the series as well as cause-effect relationships. With the CWT, we can analyze the time-series variation simply and easily address the variables' variance in a single diagram. More precisely, we use the Wavelet Power Spectrum (WPS) to analyze the similarities of variance and jumps, which may appear in the inflation and inflation uncertainty series. We further employ the Cross Wavelet Transform (XWT) to highlight the common features of the two series and their relative phase in the time-frequency space. In addition, we apply the Wavelet Coherence (WTC) together with phase-difference methods to analyze the lead-lag time-frequency relationships.

Second, we rely on the use of a novel method for the uncertainty's assessment, proposed by Chan et al. (2013). In contrast to the literature that associates inflation uncertainty with its variance and employs different generalized autoregressive conditional heteroskedasticity (GARCH)-type models, or to the literature that uses the Stock and Watson's (2007) time-varying approach, we employ the bounded model recently proposed by Chan et al. (2013), which ensures that the trend inflation lies in a specific interval (for a review of the literature on inflation uncertainty assessment, see Grimme et al., 2014). This approach proves particularly appealing for measuring uncertainty in inflation-targeting periods. Similar to the Stock and Watson's (2007) approach, the methodology is timevarying. Different from this method, however, we can apply the Chan et al.'s (2013) approach even if the states are subject to inequality constraints. Therefore, we apply this new technique and also use the Stock-Watson model for a robustness check.

Third, we examine historically the inflation-uncertainty nexus in the U.S. over 17752014, using annual data. This time-span permits an analysis of various facets of the link between inflation and its uncertainty over different exchange rate and political regimes, and over different crisis periods. Hwang (2001) also performs a historical investigation on U.S., annual data, but staring in 1926. Nevertheless, the results may change with different data frequencies. Pourgerami and Maskus (1987) state that agents adjust their expectations to changes in prices on a monthly basis; while Glezakos and Nugent (1987) hold that the use of annual or monthly data does not significantly influence the inflation-uncertainty relationship. Consequently, to assess the importance of data timing, we also use a monthly dataset over 1872:01-2015:05.

The outline of the paper is as follows. First, we present the empirical literature on the inflation-uncertainty nexus. Second, we describe Chan et al.'s (2013) approach for assessing 
inflation uncertainty and the wavelet methodology. Third, we continue with the presentation of the data and results. Finally, we conclude.

\section{Empirical literature on the inflation-uncertainty nexus}

Staring with Glezakos and Nugent (1984) and continuing with Pourgerami and Maskus (1987), a series of studies empirically investigate the inflation-inflation uncertainty nexus, stimulated by the Friedman Nobel lecture. While few studies investigate both the first and second hypotheses in Friedman's speech (i.e., Grier and Perry, 2000; Özdemir, 2010; Jiranyakul and Opiela, 2011; Bhar and Mallik, 2013; Mohd et al., 2013; Pintilescu et al., 2014), many focus on the first hypothesis, relating inflation to its uncertainty. Usually they resort to GARCH-type models to estimate the uncertainty and to Granger-causality analysis to assess the existence and direction of the causality between inflation and its uncertainty.

Most studies provide evidence in support of the Friedman-Ball hypothesis. In this line, Grier and Perry (2000) use U.S. monthly data from1948:07 to 1996:12 and GARCH-M models to estimate inflation uncertainty and find evidence in favor of the Friedman-Ball hypothesis. Similar results appear in Fountas (2001), who uses U.K. data over 1885-1998 and a GARCH method to estimate uncertainty. The positive relationship between inflation and its uncertainty also emerges in Kontonikas (2004), who employs British data over 1972-2002, and in Thornton (2008), who estimates the historical inflation-uncertainty relationship for Argentina over 1810-2005. More recently, Jiranyakul and Opiela (2011) use a bivariate constant conditional correlation generalized autoregressive conditional heteroskedastic (CCC-GARCH) specification for uncertainty and find a positive relation running from inflation to inflation uncertainty in Thailand. Jemna et al. (2014) model inflation uncertainty in Romania with a GARCH-type specification and find evidence of unidirectional Grangercausality, supporting the Friedman-Ball hypothesis. Nasr et al. (2015) uses a more complex approach to assess the inflation-uncertainty nexus. Based on a seasonal fractionally integrated smooth transition autoregressive asymmetric power GARCH and a conditional Gaussian Markov switching vector autoregressive (MS-VAR) model framework, the authors provide evidence in favor of the Friedman-Ball hypothesis for South Africa, over 1921:01-2012:12.

Different from these single-country studies, other studies analyze the inflationuncertainty relationship for a group of countries and find evidence to support the FriedmanBall hypothesis. Using an asymmetric power GARCH model for the uncertainty and data for the G7 and different emerging countries for 1987:02 to 2003:11, Daal et al. (2005) suggest 
that positive inflationary shocks affect inflation uncertainty more strongly, especially for the Latin American countries. Hartmann and Herwartz (2012) analyze the case of 22 economies from 1975:01 to 2011M5:05 and show that the effect of inflation on inflation uncertainty is more pronounced than the reverse causal effect. In the euro area countries, Caporale et al. (2012) use a time-varying AR-GARCH model for uncertainty and a VAR framework for the period 1980:01-2009:02 and show that in the euro period, the Friedman-Ball hypothesis is empirically supported. Finally, Mohd et al. (2013) report a unidirectional causality from inflationary shocks to inflation uncertainty in five ASEAN countries, using quarterly data over 1980-2011.

Another part of the literature uses GARCH-type models for assessing the inflation uncertainty and produce evidence supporting both the Friedman-Ball and CukiermanMeltzer hypotheses. The bidirectional causality between the inflation rate and its uncertainty is documented inter-alia by Thornton (2007) for 12 emerging market economies and by Fountas and Karanasos (2007) for the G7 countries. Using a nonlinear flexible regression model, Chen et al. (2008) obtain mixed results for four economies in East Asia, namely Taiwan, Hong Kong, Singapore, and South Korea. Except for Hong Kong, overwhelming statistical evidence supports the Friedman-Ball hypothesis, whereas the Cukierman-Meltzer hypothesis is validated for all four countries. Özdemir and Fisunoğlu (2008) consider the hypotheses for emerging market countries. For Jordan, Philippines, and Turkey, the authors confirm the Friedman-Ball hypothesis, and document weak evidence to support the Cukierman-Meltzer hypothesis. Similar studies are performed by Castillo et al. (2012) for Peru, by Chowdhury (2014) for India, and by Buth et al. (2015) for Cambodia, Lao PDR, and Vietnam. All support the positive relationship between inflation and its uncertainty. For developed countries, the Friedman-Ball and Cukierman-Meltzer hypotheses are confirmed by Balcilar et al. (2011). For Japan, the U.S., and the U.K., the authors show that the inflation and its uncertainty affect each other positively. Finally, for Eastern European economies, Živkov et al. (2014) use unconditional quantile regression estimation and find that both the Friedman-Ball and Cukierman-Meltzer hypotheses are confirmed.

Less empirical evidence supports the theory advanced by Pourgerami and Maskus (1987), Ungar and Zilberfarb (1993), or Holland (1995). For example, Hwang (2001) documents the negative influence of inflation on its uncertainty. Performing a historical investigation for the U.S., the author shows that inflation affected its uncertainty weakly and negatively, whereas the opposite effect is insignificant. On contrary, Payne (2008) shows that an increase in inflation uncertainty leads to a decrease in inflation in Jamaica, supporting 
Holland's stabilization-motive hypothesis (for the Bahamas and Barbados, the Friedman-Ball hypothesis is validated). Balcilar and Özdemir (2013) report similar findings for the G7 countries.

Another segment of the literature, staring with Baillie et al. (1996), finds that the inflation-uncertainty relationship leads to mixed results. Using maximum likelihood estimates of an ARFIMA-GARCH model, the authors discover that for low inflation countries such as Canada, Germany, Italy, Japan, and the U.S., no relationship exists between the conditional mean and variance of inflation, whereas for high inflation countries, strong evidence exists of joint feedback. Using GARCH models and different Granger-causality methods for the G7 countries, Grier and Perry (1998) show that in all G7 countries, inflation significantly raises inflation uncertainty. The same study, however, documents a weaker positive causality running from inflation uncertainty to inflation for Japan and France, whereas in for the U.S., the U.K., and Germany, increased inflation uncertainty lowers inflation. Conrad and Karanasos (2005) finds mixed evidence for the U.S., Japan, and the U.K. Investigating both the first and second hypotheses of Friedman (i.e., inflation causes inflation uncertainty and inflation uncertainty cause output growth), a group of studies also documents mixed evidence. Özdemir (2010) uses a VARFIMA-BEKK MGARCH model for the U.K. and finds mixed evidence regarding the effect of inflation on inflation uncertainty over the entire time-span, while no influence exists for sub-periods. Using a bivariate EGARCH model for uncertainty, Bhar and Mallik (2013) show that U.K. inflation uncertainty exerts positive effects on inflation before the inflation-targeting period. After the inflation-targeting period, the generalized impulse response functions highlight a negative influence. More recently, Pintilescu et al. (2014) test different theoretical hypothesis on the inflation-uncertainty nexus for European countries. Their results, generated by a bivariate vector autoregressive model, strongly support the Friedman-Ball hypothesis. For the other hypotheses, however, fewer significant causal relationships emerge.

The mixed evidence documented in the literature may reflect the overwhelming use of linear specifications for the employed empirical models. The relationship between the level of inflation and its uncertainty may change with monetary policy regimes changes, changes in central banks objectives, and changes in the international economic context. Therefore, the wavelet transform permits non-linearity, different lead-lag relationships, and changes in the inflation-uncertainty nexus at different cycles (i.e. periodicities). Bouoiyour and Selmi (2014) conduct such analysis for Egypt using the discrete wavelet decomposition and a battery of non-linear Granger causality tests, finding no influence in the short-run, a one-way 
causality relationship from inflation to its uncertainty in the medium-run, and bidirectional causality in the long-run. A more elegant way to assess this relationship, however, consists in the use of the CWT and its developments, which leads to easy interpretation of the results. Moreover, no previous study performs an historical analysis over such long period as we do.

\section{Methodology}

\subsection{Measuring inflation uncertainty - Chan et al.'s (2013) approach}

Frequently, researchers measure inflation uncertainty by an inflation gap, using the unobserved component framework:

$$
\pi_{t}=\tau_{t}+c_{t},
$$

where $\pi_{t}$ is the observed level of inflation with the property $\lim _{j \rightarrow \infty} E_{t}\left[\pi_{t+j}\right]=E_{t}\left[\tau_{t+j}\right]$ with the probability $1, \tau_{t}$ is the inflation trend, and $c_{t}$ is the inflation gap with the property $\lim _{j \rightarrow \infty} E_{t}\left[c_{t+j}\right]=0$ with the probability 1 .

In this framework, a constant parameter model assumes that $\tau_{t}=\tau$ and $c_{t}=\rho_{1} c_{t-1}-$ $\cdots-\rho_{p} c_{t-p}+\varepsilon_{t}$, with $\varepsilon_{t} \sim N\left(0, \sigma_{c}^{2}\right)$. In estimating the inflation trend, we resort to the unobserved components stochastic volatility model of Stock and Watson (2007), who generalize the model allowing for the inflation trend to change at varying rates over time. Thus, we can write the stochastic volatility in the innovation to the inflation trend as:

$$
\varepsilon_{t}^{T} \sim N\left(0, \exp \left(g_{t}\right)\right),
$$

where $g_{t}=g_{t-1}+\varepsilon_{t}^{g}$ and $\varepsilon_{t}^{g} \sim N\left(0, \sigma_{g}^{2}\right)$.

Compared to Stock and Watson (2007), Cogley et al. (2010) propose an alternative specification for the time-varying inflationary process without resorting to an unobserved component framework, but allowing for stochastic volatility in the state equation. They model inflation as:

$$
\pi_{t}=\emptyset_{0 t-1}+\emptyset_{1 t-1} \pi_{t-1}+\varepsilon_{t} \exp \left(\frac{h_{t}}{2}\right),
$$

where $h_{t}=h_{t-1}+\varepsilon_{t}^{h}$ and the vector of coefficients is $\emptyset_{t}=\emptyset_{t-1}+\varepsilon_{t}^{\emptyset}$.

Cogley et al. (2010) restrict the autoregressive parameter to lie inside the unit circle. Thus, the long-run mean or trend inflation $\tau_{t}=\frac{\emptyset_{0 t}}{1-\emptyset_{1 t}}$, where the future values of the vector $\varepsilon_{t}^{\emptyset}$ equal zero. The inflation gap, then, becomes:

$$
c_{t}=\tau_{t+1}-\frac{\emptyset_{0 t}}{1-\emptyset_{1 t}}
$$

with the associated model specification $c_{t+1}=\emptyset_{1 t}\left(\pi_{t}-\frac{\emptyset_{0 t}}{1-\emptyset_{1 t}}\right)+\varepsilon_{t+1} \exp \left(\frac{h_{t+1}}{2}\right)$. 
Building upon Cogley et al. (2010), Chan et al. (2013) propose an unobserved components model with an autoregression in the transitory component. Focusing on the first order autoregression case, we have the following:

$$
\left(\pi_{t}-\tau_{t}\right)=\rho_{t}\left(\pi_{t-1}-\tau_{t-1}\right)+\varepsilon_{t} \exp \left(\frac{h_{t}}{2}\right),
$$

and $\quad \tau_{t}=\tau_{t-1}+\varepsilon_{t}^{\tau}, \quad h_{t}=h_{t-1}+\varepsilon_{t}^{h}, \quad$ and $\quad \rho_{t}=\rho_{t-1}+\varepsilon_{t}^{\rho}, \quad$ where $\varepsilon_{t} \sim N(0,1)$ and $\varepsilon_{t}^{h} \sim N\left(0, \sigma_{h}^{2}\right)$.

In terms of bounding the inflation trend, the authors propose an innovation in the state equation $\varepsilon_{t}^{T} \sim T N\left(a-\tau_{t-1}, b-\tau_{t-1} ; 0, \sigma_{\tau}^{2}\right)$, where $T N\left(a, b ; \mu, \sigma^{2}\right)$ represents the Gaussian distribution with mean $\mu$ and variance $\sigma^{2}$, which are truncated to the interval $(a, b)$.

Employing the symmetry of the Gaussian distribution, the conditional expectation is given as follows:

$$
E_{t}\left[\tau_{t+1}\right]=\tau_{t}+\sigma_{\tau}\left[\frac{\emptyset\left(\frac{a-\tau_{t}}{\sigma_{t}}\right)-\emptyset\left(\frac{b-\tau_{t}}{\sigma_{\tau}}\right)}{\Phi\left(\frac{b-\tau_{t}}{\sigma_{\tau}}\right)-\Phi\left(\frac{a-\tau_{t}}{\sigma_{\tau}}\right)}\right], \text { if } a \leq \tau_{t} \leq b
$$

For the inflation gap $\left(\rho_{t}\right)$, the innovation is $\varepsilon_{t}^{\rho} \sim T N\left(a_{\rho}-\rho_{t-1}, b_{\rho}-\rho_{t-1} ; 0, \sigma_{\rho}^{2}\right)$, while the conditional expectation becomes:

$$
E_{t}\left[\rho_{t+1}\right]=\rho_{t}+\sigma_{\rho}\left[\frac{\emptyset\left(\frac{a_{\rho}-\rho_{t}}{\sigma_{\rho}}\right)-\emptyset\left(\frac{b_{\rho}-\rho_{t}}{\sigma_{\rho}}\right)}{\Phi\left(\frac{b_{\rho}-\rho_{t}}{\sigma_{\rho}}\right)-\Phi\left(\frac{a_{\rho}-\rho_{t}}{\sigma_{\rho}}\right)}\right] \text {, if } a_{\rho} \leq \rho_{t} \leq b_{\rho}
$$

For the inflation gap, the bounds are generated to satisfy the zero convergence condition of the gap in the long-run. Therefore, we limit $\rho_{t}$ to lie inside the unit circle or within constant limits (i.e., $0<\rho_{t}<1$ ).

\subsection{Wavelet analysis}

The wavelet transform maps a time-dependent function from its original representation into an alternative representation in the time-scale domain by recursively applying two wavelet filters, namely the father wavelets $\int \phi(t) d t=1$ (associated with low-frequencies and trend components) and the mother wavelets $\int \psi(t) d t=0$ (associated with high-frequency detail components).

Different classes of wavelet transforms exist, which apply directly for financial data, but the CWT possesses simplicity in the interpretation of the results in a single diagram. The CWT provides an alternative approach to short-term Fourier transforms, which overcomes resolution problems. We now provide a brief description of the CWT and its developments.3.2.1. The continuous wavelet transform 
The wavelets are localized both in time and frequency $\psi_{\tau, s}(t)$, where $\tau$ is time position, and $s$ is the scale (Rua and Nunes, 2009):

$$
\psi_{\tau, s}(t)=\frac{1}{\sqrt{s}} \psi\left(\frac{t-\tau}{s}\right),
$$

where $\frac{1}{\sqrt{s}}$ ensure the comparability of wavelet transforms across scales and time.

According to Gençay et al. (2002), a mother wavelet, $\psi(t)$, must fulfill several conditions. The admissibility condition allows the reconstruction of a time series $x(t)$ from its continuous wavelet transform $W_{x}(\tau, s)$, as follows:

$$
x(t)=\frac{1}{C_{\psi}} \int_{-\infty}^{+\infty}\left[\int_{-\infty}^{+\infty} \frac{1}{\sqrt{s}} \psi\left(\frac{t-\tau}{s}\right) W_{x}(\tau, s) d \tau\right] \frac{d s}{s^{2}}
$$

Thus, the CWT of a time series $x(t), t=1, \ldots, N$ is:

$$
W_{x}(\tau, s)=\frac{1}{\sqrt{s}} \sum_{t=1}^{N} x(t) \psi^{*}\left(\frac{t-\tau}{s}\right)
$$

The use of the Fourier space represents a common practice for the wavelet transform (see Torrence and Compo, 1998), while the Morlet wavelet (with $\omega_{0}=6$ ) is considered a good choice for decomposing financial time series. It can be defined as follows:

$$
\psi(t)=\pi^{-\frac{1}{4}} e^{i \omega_{0} t} e^{-\frac{1}{2} t^{2}}
$$

where $t$ is the time-dimension and $\omega_{0}$ is the frequency-dimension.

Consequently, the corresponding Fourier transform becomes:

$$
\hat{\psi}(\omega)=\pi^{\frac{1}{4}} \sqrt{2 e}^{-\frac{1}{2}\left(\omega-\omega_{0}\right)^{2}} .
$$

\subsubsection{The wavelet power spectrum}

The wavelet power spectrum provides information about similarities between series' variances and detects structural breaks. The wavelet power is $\left|W_{x}(\tau, s)\right|^{2}$, where the complex argument of $W_{x}(\tau, s)$ represents the local phase. Because the wavelet is not completely localized in time, the CWT contains edge artifacts that questions the quality of results at the borders of the diagram. The cone of influence (COI) is used to show where we cannot ignore the edge effects. 
The white-noise and red-noise wavelet power spectra, from which the corresponding distribution at each time $n$ and scale $s$ is derived under the null, is (Torrence and Compo, 1998):

$$
D\left(\frac{\left|W_{x}(\tau, s)\right|^{2}}{\sigma_{x}^{2}}<p\right)=\frac{1}{2} P_{k} \chi_{v}^{2}(p),
$$

where $v$ equals 1 for real wavelets and 2 for complex ones.

\subsubsection{The cross-wavelet transform}

The XWT of two time series $x_{t}$ and $y_{t}$ is defined as $W_{x y}=W_{x} W_{y^{*}}$, where $W_{x}$ and $W_{y}$ are the

wavelet transforms. The cross-wavelet power becomes $\left|W_{x y}\right|$, while the complex argument $\arg W_{x y}$ is the local relative phase between $x_{t}$ and $y_{t}$ in the time-frequency space. The theoretical distribution of the XWT of two time series is (Torrence and Compo, 1998):

$$
D\left(\frac{\left|W_{x t}(s) W_{y t} *(s)\right|}{\sigma_{x} \sigma_{y}}<p\right)=\frac{z_{v}(p)}{v} \sqrt{P_{x k} P_{y k}},
$$

where $z_{v}(p)$ is the confidence level and $*$ denotes complex conjugation.

\subsubsection{The wavelet coherence}

If the XWT can observe the co-movements in two time series, then it is not clear which variable causes the other. Therefore, we compute the WTC as the ratio of the cross-wavelet spectrum to the product of the spectrum of each series. We treat this as the local correlation of the two series, both in time and frequency. Torrence and Webster (1999) define the WTC of two time series with $W_{x}(\tau, s)$ and $W_{y}(\tau, s)$ wavelet transforms as follows:

$$
R^{2}(\tau, s)=\frac{\left|S\left(s^{-1} W_{x y}(\tau, s)\right)\right|^{2}}{S\left(s^{-1}\left|W_{x}(\tau, s)\right|^{2}\right) \cdot S\left(s^{-1}\left|W_{y}(\tau, s)\right|^{2}\right)}
$$

where $S($.$) is the smoothing operator and s$ is the wavelet scale.

\subsubsection{The cross-wavelet phase angle}

To establish the confidence interval of the phase difference, we use the circular mean of the phase over regions with greater than $5 \%$ statistical significance (i.e., outside/above the COI) defined as: 


$$
a_{m}=\arg (x, y), \text { with } x=\sum_{t=1}^{n} \cos \left(a_{t}\right) \text { and } y=\sum_{t=1}^{n} \sin \left(a_{t}\right) .
$$

The scatter of angles around the mean gives the confidence interval based on the circular standard deviation $s=\sqrt{-2 \ln (R / t)}$, where: $R=\sqrt{x^{2}+y^{2}}$.

\section{Data and results}

\subsection{Data}

We measure inflation using Consumer Price Index (CPI) data. The annual CPI data, covering 1774 to 2014 with a base year of 1982-1984, comes from Professor Sahr's website (http://oregonstate.edu/cla/polisci/sahr/sahr). We also download monthly CPI data, covering 1871:01-2015:05 with a base year of 1982-1984, from Professor Shiller's website (http://www.econ.yale.edu/ shiller/data.htm). We compute inflation is computed as year-onyear percent change, hence the inflation data cover1775-2014 and 1872:01-2015:05. We compute the inflation uncertainty, using the Chan et al.'s (2013) approach (Uncertainty 1).

For robustness purposes, however, we also resort to the Stock and Watson (2007) method for calculating inflation uncertainty (Uncertainty 2), as shown in the Appendices. Figure 1 presents the (year-on-year) inflation rates for annual and monthly data, and also the corresponding (standardized to a unit standard deviation) measures of uncertainties based on the methods proposed by Chan et al., (2013, Uncertainty 1) and Stock and Watson (2007, Uncertainty 2). Clearly, as observed from Figures $1 \mathrm{a}$ and $1 \mathrm{~b}$, the inflation rate achieved much more volatility near the beginning of the sample with inflation rates varying between negative and positive values. The inflation rate exhibits primarily positive values since the 1950 s with peaks around the two oil shocks in 1973 and 1979, but with relatively smaller volatility than near the beginning of the sample. Figures $1 \mathrm{c}$ and 1d illustrate that the uncertainty measures under both approaches declined over time, with the Stock and Watson (2007) approach generally generating higher volatility. ${ }^{2}$

\subsection{Empirical findings}

\subsubsection{Results obtained using annual data}

\footnotetext{
${ }^{2}$ For the annual data, the correlation between the two measures of uncertainty is 0.8758 , while that for the monthly data is 0.6264 , with both being statistically significant at the 1 percent level. Interestingly, a causality analysis reveals that for the annual data Uncertainty 2 leads Uncertainty 1, while for the monthly data, the causality runs both ways. The causality results are robust to alternative choices of lag-length. In addition, when we repeat the causality test at the annual frequency over 1872 to 2014 to correspond with the sample of the monthly frequency data, we still observe that Uncertainty 2 leads Uncertainty 1. Complete details of these results are available from the authors on request.
} 
The WPS of the inflation and its uncertainty is presented in a contour plot with three dimensions: time, frequency and color code. The blue color of the power spectrum associates with low power, while the red color, with high power. The thin black line borders identify the cone of influence, and show the edge effects that arise from boundary conditions imposed to datasets with finite lengths. Thus, the area below the COI shows no statistical influence.

Figure 2 shows that the inflation series exhibits strong variability in the short-run (1-4year cycle) at the beginning of the 1810s (the end of the international restrictions on the U.S. commerce), but also during the Great Depression. In the medium-run (8-16-year cycle), islands of higher variability occur around 1800, during the 1860s, and during the 1930s, while in the long-run (16-32-year cycle), we observe relatively strong variability from just before WWI through the entire interwar period and ending around 1960.

We can associate the medium-run variability in the inflation rate registered during the 1860s with the U.S. Civil War, while that during the 1930s with the Great Depression. In contrast, inflation uncertainty series present no important variability in the short-run, while in the medium- and long-runs, we notice some variability at the begging of 1800s (an era characterized by a strong incertitude in U.S. international trade).

That is, the WPS offers no precise information about the historical inflation-uncertainty relationship in the U.S. Because the similarities between the portrayed patterns of the two series are reduced, and it is therefore difficult to determine if this is merely a coincidence, we proceed to the XWT and WTC computation, which may provide clarification (Figure 2).

Several conclusions emerge from Figure 3. First, the XWT (Figure 3a) shows that in the short-run, the arrows orient in all directions, thus, confirming all the theoretical hypothesis describe above. The color spectrum, however, illustrates that a weak relationship exists between variables in the short-term (similar to the results reported for Egypt by Bouoiyour and Selmi, 2014). In the medium- and long-runs (4-32-year cycle), however, the variables move in-phase with each other. That is, the arrows point to the right and down, meaning that inflation uncertainty leads the inflation level, supporting the CukiermanMeltzer hypothesis. The results refute the Friedman-Ball hypothesis, in contrast to the large majority of studies that document a bidirectional positive causal relationship between inflation and its uncertainty. The results confirm the Pourgerami-Maskus (1987) and UngarZilberfarb (1993) hypothesis only for short periods during the 1820s and 1920s, when prices stabilize in the U.S., and only in the short-run (1-4-year cycle).

Second, because the XWT describes the common power of two series without normalisation to a single wavelet power spectrum, it may generate misleading results, as it 
multiplies the continuous wavelet transform of two time series. Therefore, to assess the leadlag relationship, we resort to the WTC, which shows both the frequency bands and the time intervals where we observe strong co-variance (Figure $3 b$ ).

The results provide mixed evidence for the short-run (1-4-year cycle). First, during the 1810s, the arrows point left and up, showing that the variables are out-of-phase and inflation lags its uncertainty. Thus, these results confirm the Holland's (1995) stabilization hypothesis, when the Congress did not approve the renewal of the Second Bank of the United States. Second, the inflation level negatively influences its uncertainty during the 1830 s and the 1920s, providing supporting evidence for the Pourgerami-Maskus (1987) and UngarZilberfarb (1993) hypothesis, which shows a strong anti-inflation incentive for the authorities. Third, during the 1860s, high inflation caused by the Civil War causes the arrows to point to the right and up at the 4-6-year cycle, meaning that the variables are in-phase and inflation leads, supporting the Friedman-Ball hypothesis.

In the medium- and long-runs (8-32-year cycle), however, we notice co-movements from 1850 up to 1950 . In all cases, the arrows point right and down, again sustaining the Cukierman-Meltzer hypothesis. This means that the U.S. government at the beginning of the 20th century put greater emphasis on growth and employment than on price stability.

All in all, the results show a complex relationship between inflation and its uncertainty in the short-run, while in the long-run, uncertainty proves decisive for explaining inflation. Figure 2 also shows less evidence of this effect during more recent years, due to the progress in inflation forecasting that reduces its uncertainty.

The results, however, may depend on how we measure inflation uncertainty. Therefore, to consider the robustness of our findings, we proceed to an alternative analysis, resorting to the Stock-Watson (2007) measure of uncertainty (Appendix A reports the results). We can make two observations. First, the WPS produces more similarities between inflation and its uncertainty (Uncertainty 2), especially during the interwar period. Second, the WTC documents similar outcomes in the short-run, confirming the mixed evidence reported by the previous literature. In the long-run (8-32-year of cycle), the variables remain in-phase. In this case, however, inflation leads uncertainty, thus, supporting the Friedman-Ball hypothesis. We can rationalize these results as follows: In the case of unbounded inflation rates, an increased price level fosters market uncertainty. On contrary, if the inflation and its trend are bounded (the argument of Chan et al. (2013) for the Volcker reform), then uncertainty leads inflation in the medium- and long-runs. 


\subsubsection{Results obtained using monthly data}

The results may also depend on the frequency of the data used for estimation. Pourgerami and Maskus (1987) argue that rational expectations are formed on a monthly basis. Therefore, we now consider U.S. monthly statistics over the period 1872:01-2015:05.

Figure 4 presents the WPS of the two series. As expected, the series present stronger variance in the short-run (1-4-year cycle) as compared to the annual data, especially before the 1950s. In fact, the annual data hides substantial effects revealed in the monthly data for the uncertainty measure in the short-, medium-, and long-runs (1-to-32-year cycle). In addition the inflation effects become more intense when comparing the monthly to the annual outcomes.

This stronger variability in inflation and its uncertainty is explained by a number of factors - the Federal Reserve System did not exist prior to 1913, the interwar period saw an attempt to return to the Gold Standard, and WWI and WWII prompted the Treasury to finance the war efforts with low interest rates. The adoption of the Treasury-Federal Reserve Accord of 1951 relieved the Federal Reserve from keeping the interest cost of the federal debt low. We also notice a particular situation for the uncertainty series, where a strong variation occurs in the medium-run from 1975 to 1985 following the 1973 OPEC oil crisis.

Figure 4 highlights the XWT and WTC analysis for monthly data. The XWT results (Figure 5a) confirm the high variability registered by the two monthly time-series before 1950. Differing from the annual results, however, the monthly data reveal in the short- and medium-runs (1-16-year cycle) that the arrows point both to the left and right, indicating that the variables can remain either in-phase or out-of-phase.

The WTC analysis (Figure 5b) shows noteworthy co-movements in the very short-run (0.25-0.5-years scale) for monthly data. As expected, the results are mixed, supporting either the Pourgerami-Maskus (1987) and Ungar-Zilberfarb (1993) hypothesis for the 1930s, or supporting the Friedman-Ball hypothesis for the 1970s. For the 1-4-year cycle, a decrease in inflation increases its uncertainty after the National Banking Act of 1863, during a period characterized by strong financial incertitude. We also notice a similar relationship in the 1920s, when the Fed starts using open market operations as a monetary policy tool. In the medium-run (4-8-year cycle), the results provide evidence that supports the Friedman-Ball hypothesis for 1965 to 1985 (similar to the findings using annual data).

If we rely on the Stock-Watson (2007) measure of inflation uncertainty (Appendix B), we see that the WPS presents similar results as those found in Chan et al.'s (2013). For the WTC analysis, however, we notice slight differences in the short- and medium-runs (1-16- 
year cycle). This time, at the beginning of the $20^{\text {th }}$ century, the results provide counterevidence against the Friedman-Ball hypothesis and support for the CukiermanMeltzer hypothesis. The Friedman-Ball hypothesis is, however, validated from WWII until 1970. In the long-run (16-32-year cycle), we can notice common movements during the interwar period until 1960, where the Cukierman-Meltzer hypothesis prevails.

We conclude that in the short-run, mixed evidence exists regarding the inflationuncertainty nexus in the U.S. In addition, the co-movements are stronger before WWII. Further, in the medium- and long-runs, a positive relationship exists between the variables. But the validation of the Friedman-Ball or the Cukierman-Meltzer hypothesis depends on how we measure uncertainty. Finally, noteworthy differences appear between the two datasets. First, as mentioned above, in the short-run (1-4-year cycle) the use of monthly data reveals strong co-movements around WWII, which does not occur with the annual data. Second, in the medium-run (4-8-year cycle), the annual data indicate strong co-movements over 1880 to 1890 , which does not occur for the monthly data. Finally, in the long-run (8-16year cycle), the annual data show a strong relationship between the inflation and its uncertainty from 1880 through the 1940s, while the use of monthly data highlights strong comovements over 1970 to 1990, supporting the Friedman-Ball hypothesis. This evidence supports the argument advanced by Pourgerami and Maskus (1987), underlining the importance of the frequency of the data.

\section{Conclusions}

Starting with Friedman's (1977) theory about the real effects of inflation, economists have generated mixed empirical evidence as to the relationship that exists between inflation and its uncertainty. A plethora of studies investigate this nexus, relying of various approaches to measure uncertainty, including different linear or non-linear causal models to accurately represent the relationship between inflation and its uncertainty.

Considering the U.S. case, we tackle four questions in this paper. First, we consider how the inflation-uncertainty nexus evolves over time and frequencies over a long time horizon. Second, we examine how different measures of uncertainty affect our results. Third, we test the effects of data-frequency on the inflation-uncertainty relationship. Fourth, we perform an historical investigation of this relationship for over a century's worth of annual data and nearly a centuries worth of monthly data. Note that the Federal Reserve came into 
existence in 1913. Thus, for portions of our samples, a central bank, the Federal Reserve, did not exist.

We show that both data frequency and the measurement of inflation uncertainty generate significant differences in the interpretation of the inflation-uncertainty nexus in the medium and long runs. More precisely, when we use a bounded measure of uncertainty, the results point generally support the Cukierman-Meltzer hypothesis, while when we use an unobserved-components stochastic-volatility model, where the innovation variance varies with time, we generally find support for the Friedman-Ball hypothesis. The interdependences emerge most strongly during and after financial distress periods. In the short-run, however, the findings highlight mixed evidence, providing an explanation for the mixed results documented in the prior empirical literature.

While this study focuses on the inflation-uncertainty nexus covering the second part of Friedman's (1977) hypothesis, we can extend the analysis toward the effect of inflation uncertainty on real economic activity, covering both the first and the second reasoning by Friedman. For this purpose, we can use the wavelet partial coherency, testing the first reasoning while making abstraction of the output growth influence, and the second reasoning, eliminating the influence of inflation. 


\section{References}

Baillie, R., Chung, C., Tieslau, M., 1996.Analyzing inflation by the fractionally integrated ARFIMA-GARCH model. Journal of Applied Econometrics, 11, 23-40.

Balcilar, M., Özdemir, Z.A., Cakan, E., 2011.On the nonlinear causality between inflation and inflation uncertainty in the G3 countries. Journal of Applied Economics, XIV, 269-296.

Balcilar, M., Özdemir, Z.A., 2013.Asymmetric and time-varying causality between inflation and inflation uncertainty in G-7 countries. Scottish Journal of Political Economy, 60, $1-41$.

Ball, L., 1992. Why does high inflation raise inflation uncertainty. Journal of Monetary Economics, 29, 371-388.

Bhar, R., Mallik, G., 2013. Inflation uncertainty, growth uncertainty, oil prices, and output growth in the UK. Empirical Economics, 45, 1333-1350.

Bouoiyour, J., Selmi, R., 2014. The Nexus between Inflation and Inflation Uncertainty via wavelet approach: Some Lessons from the Egyptian case. Economics Bulletin, 34, 2093-2106.

Buth, B., Kakinaka, M., Miyamoto, H., 2015. Inflation and inflation uncertainty: The case of Cambodia, Lao PDR, and Vietnam. Journal of Asian Economics, 38, 31-43.

Caporale, G.M., Onorante, L., Paesani, P., 2012. Inflation and inflation uncertainty in the euro area. Empirical Economics, 43, 597-615.

Castillo, P., Humala, A., Tuesta, V., 2012. Regime shifts and inflation uncertainty in Peru. Journal of Applied Economics, XV, 71-87.

Chan, J.C.C., Koop, G., Potter, S.M., 2013. A New Model of Trend Inflation. Journal of Business \& Economic Statistics, 31, 94-106.

Chen, S.-W., Shen, C.-H., Xie, Z., 2008. Evidence of a nonlinear relationship between inflation and inflation uncertainty: the case of the four little dragons. Journal of Policy Modeling, 30, 363-376.

Chowdhury, A., 2014. Inflation and inflation-uncertainty in India: the policy implications of the relationship. Journal of Economic Studies, 41, 71-86.

Cogley, T., Primiceri, G. and Sargent, T., 2010. Inflation-gap persistence in the U.S. American Economic Journal: Macroeconomics, 2, 43-69.

Conrad, C., Karanasos, M., 2005. On the inflation uncertainty hypothesis in the USA, Japan, and the UK: a dual long memory approach. Japan and the World Economy, 17, 327-343. 
Cukierman, A., Meltzer, A., 1986. A theory of ambiguity, credibility, and inflation under discretion and asymmetric information. Econometrica, 54, 1099-1128.

Daal, E., Naka, A., Sanchez, B., 2005. Re-examining inflation and inflation uncertainty in developed and emerging countries. Economics Letters, 89, 180-186.

Fountas, S., 2001. The relationship between inflation and inflation uncertainty in the UK: 1885-1998. Economics Letters, 74, 77-83.

Fountas, S., Karanasos, M., 2007. Inflation, output growth, and nominal and real uncertainty: empirical evidence for the G7. Journal of International Money and Finance, 26, 229250.

Friedman, M., 1968.The role of monetary policy. American Economic Review, 58, 1-17.

Friedman, M., 1977. Nobel lecture: inflation and unemployment. Journal of Political Economy, 85, 451-472.

Frohman, D.A., Leroy, O.L., Willet, T.D., 1981. Uncertainty Costs of High Inflation. Federal Reserve Bank of Dallas Voice, 1-9.

Gençay, R., Selçuk, F.,Whitcher, B., 2002.An Introduction to Wavelets and Other Filtering Methods in Finance and Economics. San Diego: Academic Press.

Glezakos, C., Nugent, J.B., 1984. Price instability and inflation: The Latin American case. World Development, 12, 744-758.

Glezakos, C., Nugent, J.B., 1987. The relationship between the rate of inflation and its unpredictability in high inflation Latin American Countries. World Development, 15, 291-293.

Grier, K.B., Perry, M.J., 1998. On inflation and inflation uncertainty in the G7 countries. Journal of International Money and Finance, 17, 671-689.

Grier, K.B., Perry, M.J, 2000. The effects of real and nominal uncertainty on inflation and output growth: some GARCH-M evidence. Journal of Applied Econometrics. 15, 45-58.

Grimme, C., Henzel, S.R., Wieland, E., 2014. Inflation uncertainty revisited: a proposal for robust measurement. Empirical Economics, 47, 1497-1523.

Hartmann, M., Herwartz, H., 2012.Causal relations between inflation and inflation uncertainty-Cross sectional evidence in favour of the Friedman-Ball hypothesis. Economics Letters, 115, 144-147.

Holland, S., 1995. Inflation and uncertainty: tests for temporal ordering. Journal of Money, Credit and Banking, 27, 827-837.

Hwang, Y., 2001. Relationship between inflation rate and inflation uncertainty. Economics Letters, 73, 179-186. 
Jemna, D.V., Pintilescu, C., Viorică, E.D., Asandului, M., 2014.Inflation and inflation uncertainty in Romania. Economic Computation \& Economic Cybernetics Studies \& Research, 48, 1-19.

Jiranyakul, K., Opiela, T.P., 2011. The impact of inflation uncertainty on output growth and inflation in Thailand. Asian Economic Journal, 25, 291-307.

Kim, C.-J., 1993. Unobserved-component time series models with Markov-switching heteroskedasticity: changes in regime and the link between inflation rates and inflation uncertainty. Journal of Business and Economic Statistics, 11, 341-349.

Kontonikas, A., 2004. Inflation and inflation uncertainty in the United Kingdom, evidence from GARCH modelling. Economic Modelling, 21, 525-543.

Mohd, S.H., Baharumshah, A.Z., Fountas, S., 2013. Inflation, inflation uncertainty and output growth: Recent evidence from ASEAN-5 countries. The Singapore Economic Review, $58,1-17$.

Nasr, A.B., Balcilar, M., Ajmi, A.N., Ayed, G.C., Gupta, R., van Eyden, R., 2015. Causality between inflation and inflation uncertainty in South Africa: Evidence from a Markovswitching vector autoregressive model. Emerging Markets Review, 24, 46-68.

Okun, A., 1971. The mirage of steady inflation. Brookings Papers on Economic Activity, 2, 485-498.

Özdemir, Z.A., 2010. Dynamics of inflation, output growth and their uncertainty in the UK: an empirical analysis. The Manchester School, 78, 511-537.

Özdemir, Z.A., Fisunoğlu, M., 2008. On the inflation-uncertainty hypothesis in Jordan, Philippines and Turkey: a long memory approach. International Review of Economics and Finance, 17, 1-12.

Payne, J.E., 2008. Inflation and inflation uncertainty: evidence from the Caribbean region. Journal of Economic Studies, 35, 501-511.

Phelps, E. S., 1967. Phillips curves, expectations of inflation and optimal employment over time. Economica, 34, 254-281.Pintilescu, C, Jemna, D.V., Viorică, E.D., Asandului, M., 2014. Inflation, Output Growth, and Their Uncertainties: Empirical Evidence for a Causal Relationship from European Emerging Economies. Emerging Markets Finance \& Trade, 50, 78-94.

Pourgerami, A., Maskus, K., 1987. The effects of inflation on the predictability of price changes in Latin America: some estimates and policy implications. World Development, 15, 287-290. 
Rua, A, Nunes, L.C., 2009.International comovement of stock market returns: a wavelet analysis. Journal of Empirical Finance, 16, 632-639.

Stock, J., Watson, M., 2007. Why has U.S. inflation become harder to forecast? Journal of Money, Credit and Banking, 39, 3-33.

Thornton, J., 2007. The relationship between inflation and inflation uncertainty in emerging market economies. Southern Economic Journal,73, 858-870.

Thornton, J., 2008. Inflation and inflation uncertainty in Argentina, 1810-2005.Economics Letters, 98, 247-252.

Torrence, C., Compo, G.P., 1998. A practical guide to wavelet analysis. Bulletin of the American Meteorological Society, 79, 61-78.

Torrence, C., Webster, P.J., 1999. Interdecadal Changes in the ENSO-Monsoon System. Journal of Climate, 12, 2679-2690.

Ungar, M., Zilberfarb, B-Z., 1993. Inflation and its unpredictability-theory and empirical evidence. Journal of Money, Credit and Banking, 25, 709-720.

Živkov, D., Njegić, J., Pećanac, M., 2014.Bidirectional linkage between inflation and inflation uncertainty - the case of Eastern European countries. Baltic Journal of Economics, 14, 124-139.

Figure 1. Annual and Monthly Data Plots

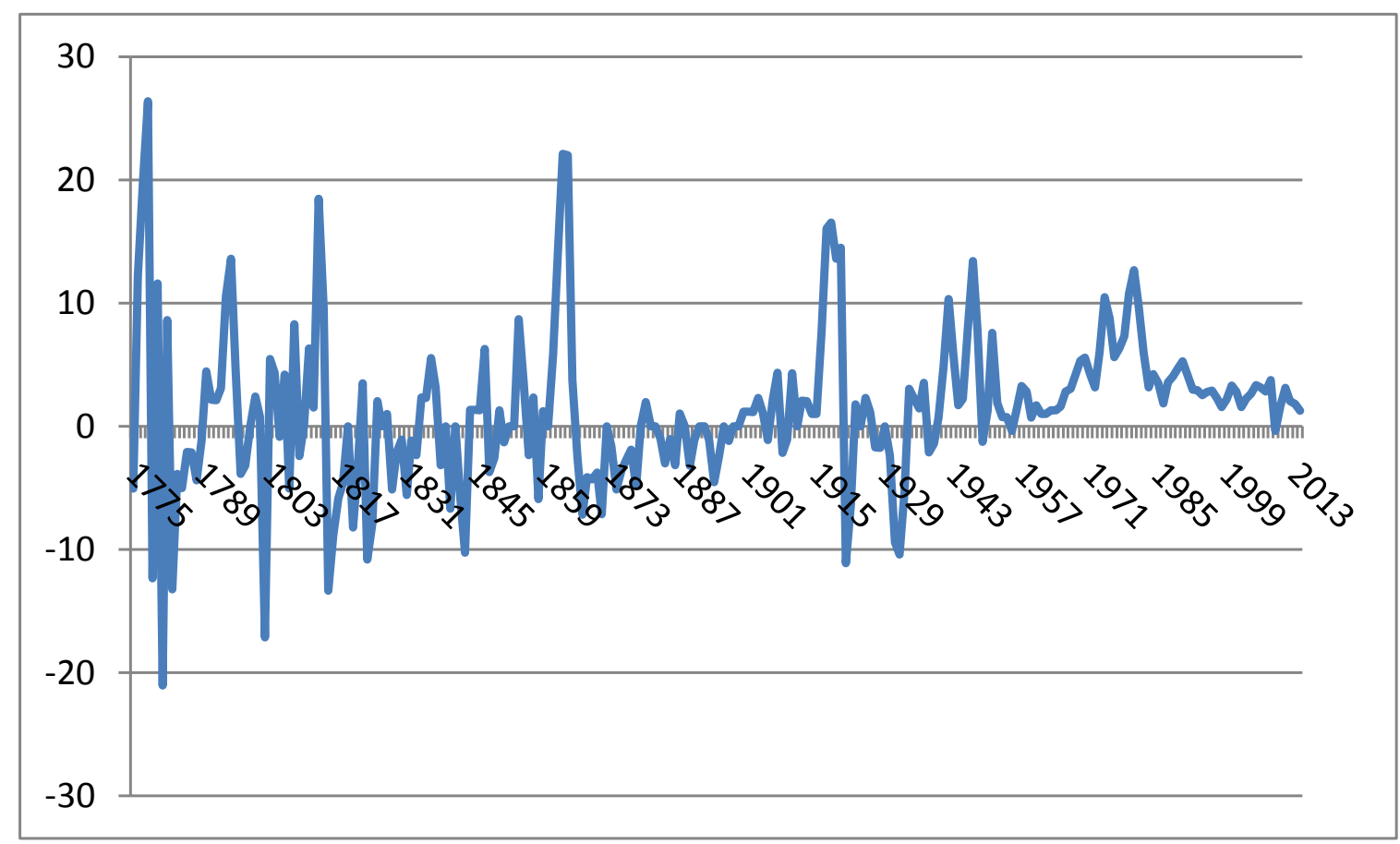

Figure 1a. Annual Inflation Rate (1775-2014) 


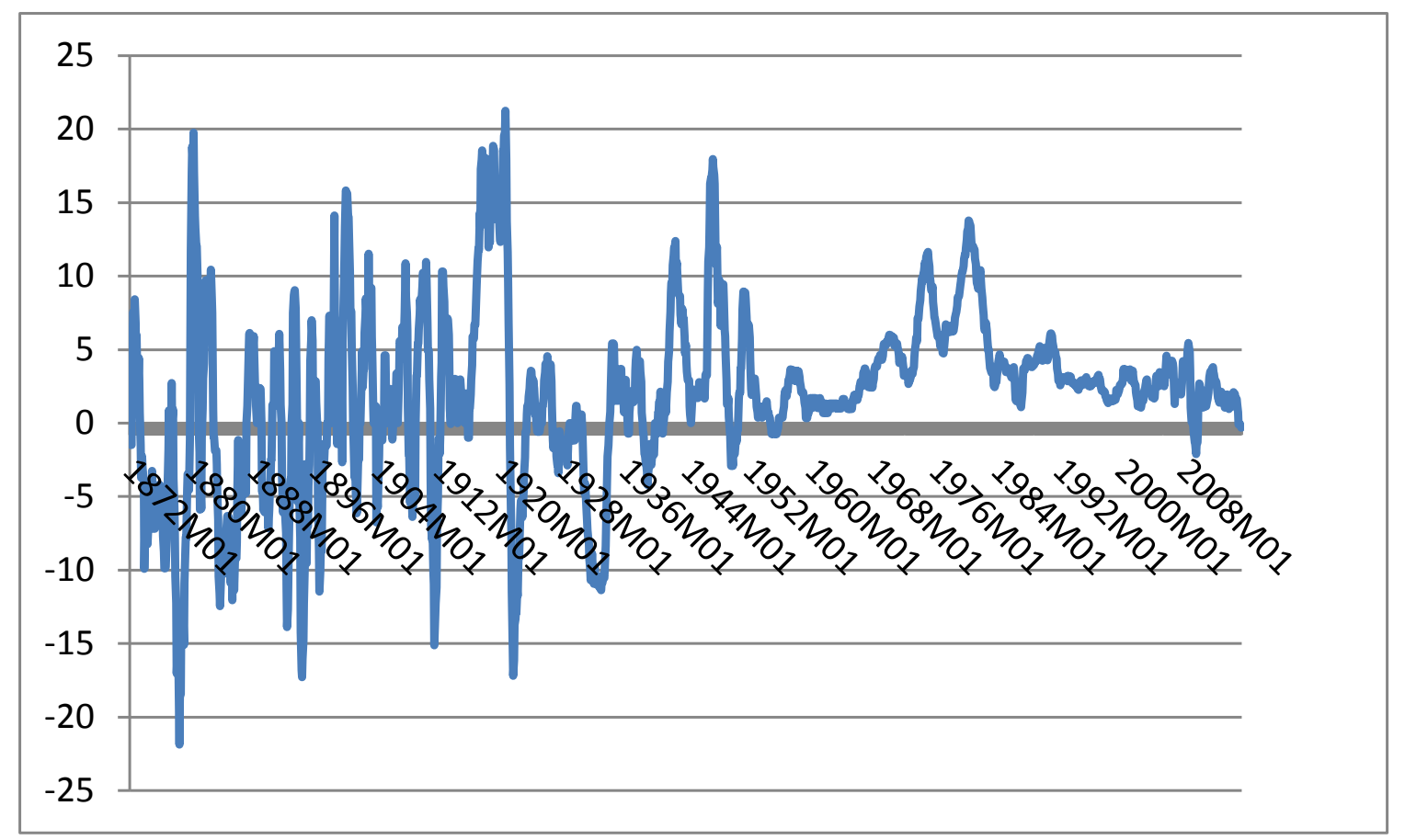

Figure 1b. Year-on-Year Monthly Inflation Rate (1872:01-2015:05)

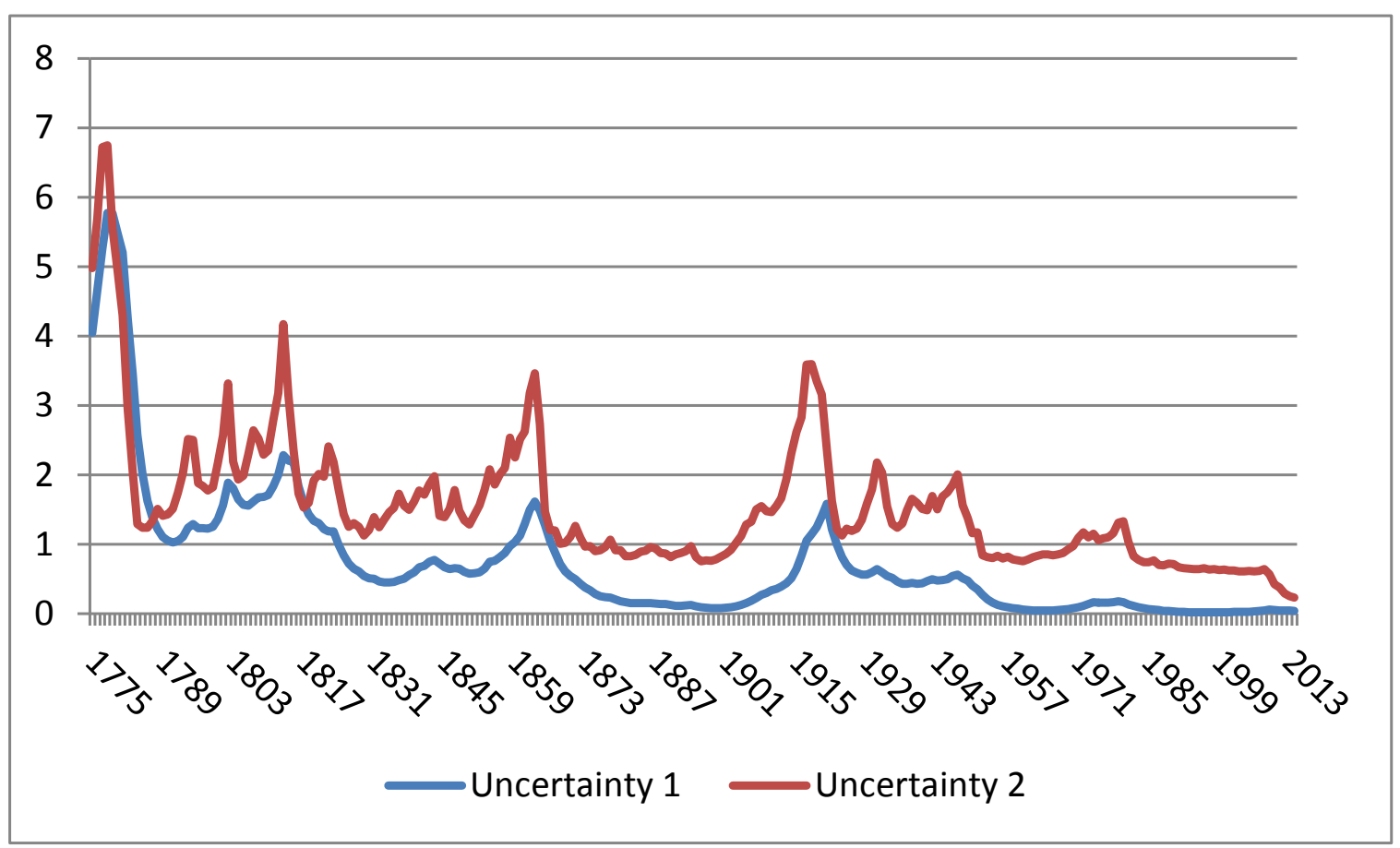

Figure 1c. Measures of Annual Inflation Uncertainties (1775-2014) 


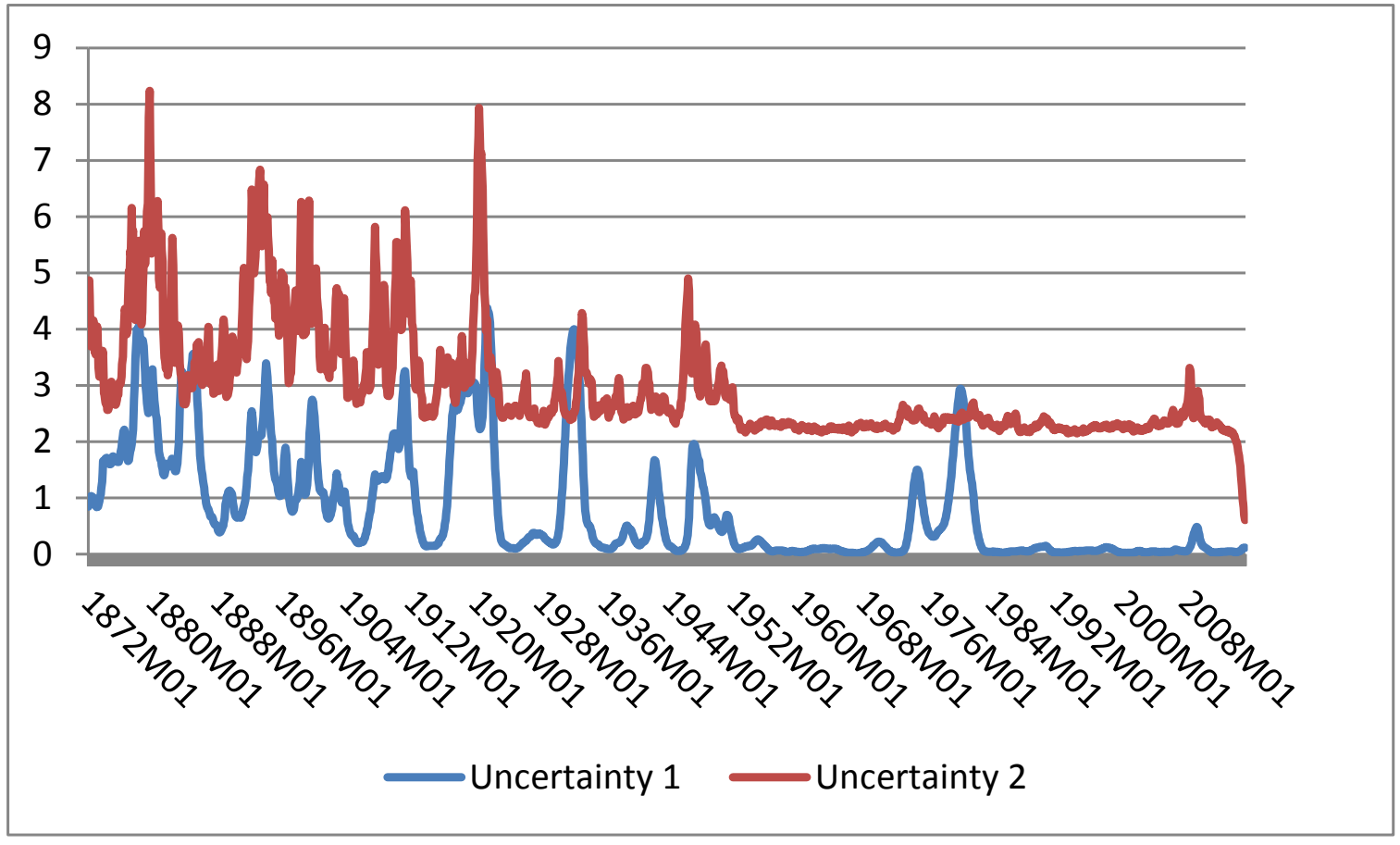

Figure 1d. Measures of Monthly Inflation Uncertainties (1872:01-2015:05) 

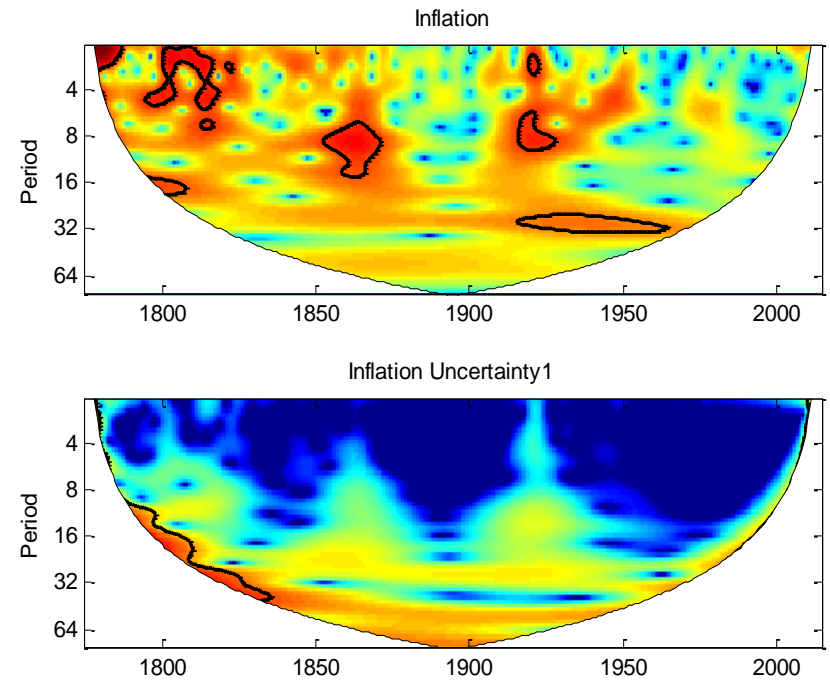

Notes: The black contour designates the 5\% significance level. $Y$ axis measures frequencies or scales, from the shortest scale (1-year cycle) to the longest scale (64-years cycle). X-axis represents the time period, covering the interval 1775 to 2014.

Figure 2. The WPS of the inflation series and its uncertainty (annual data)

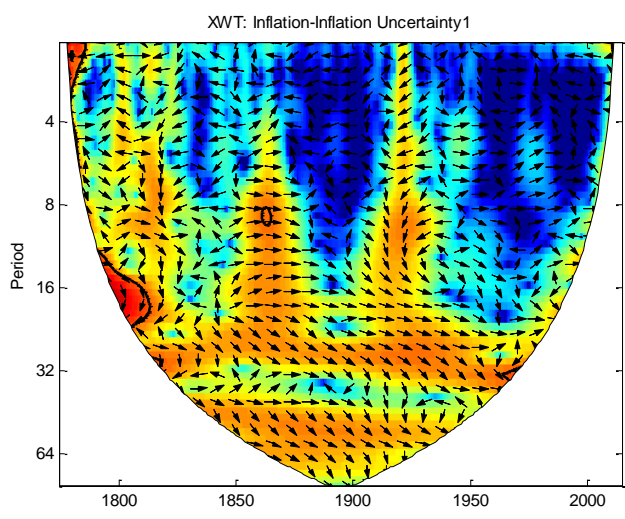

(a)

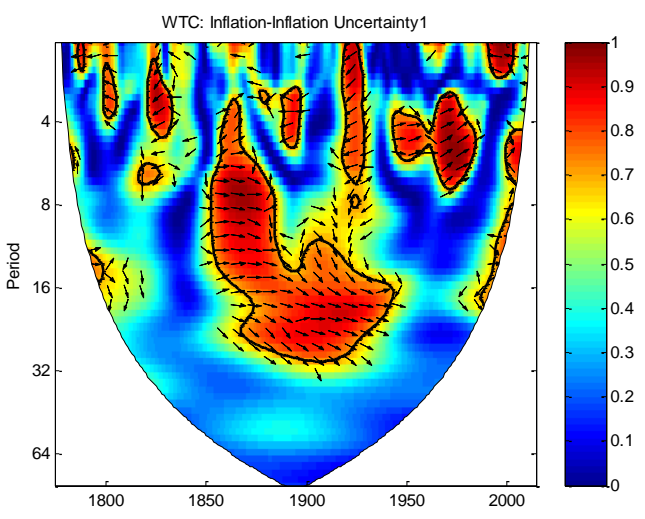

(b)

Notes: The black contour designates the 5\% significance level. $Y$-axis measures frequencies or scales, from the shortest scale (1-year cycle) to the longest scale (64-years cycle). X-axis represents the time period, covering the interval 1775 to 2014. The phase differences between the two series are indicated by arrows. Arrows pointing to the right mean that the variables are in-phase (they increase and decrease together), to the right and up mean that the inflation is leading, and to the right and down mean that the inflation is lagging. Arrows pointing to the left mean that the variables are out-of-phase, to the left and up mean that the inflation is lagging and to the left and down mean that the inflation is leading.

Figure 3. The XWT of the inflation series and its uncertainty (annual data) 

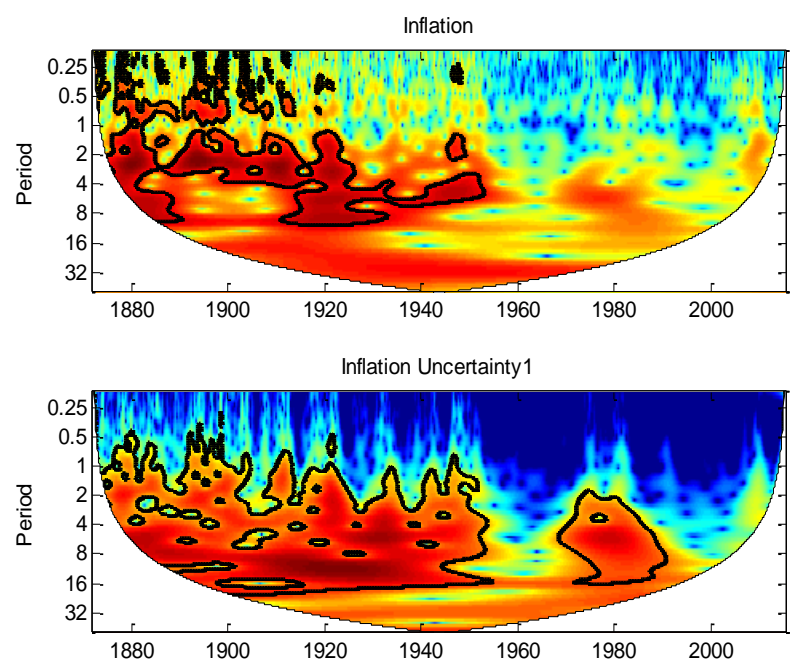

Notes: The black contour designates the 5\% significance level. Yaxis measures frequencies or scales, from the shortest scale (1-year cycle) to the longest scale (32-years cycle). X-axis represents the time period, covering the interval 1872:01 to 2015:05.

Figure 4. The WPS of the inflation series and its uncertainty (monthly data)

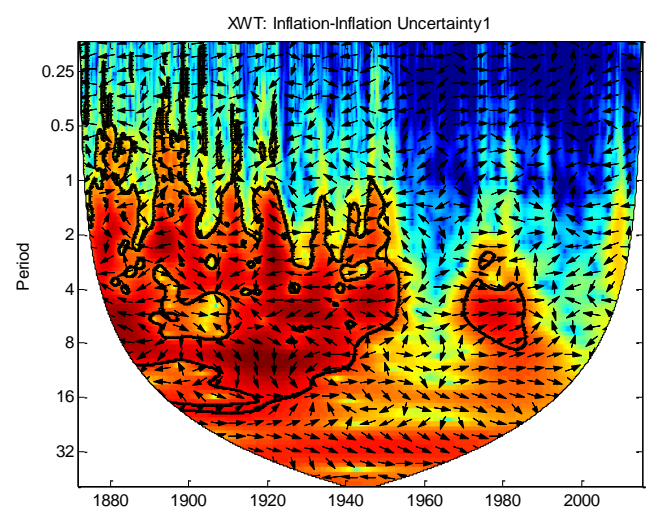

(a)

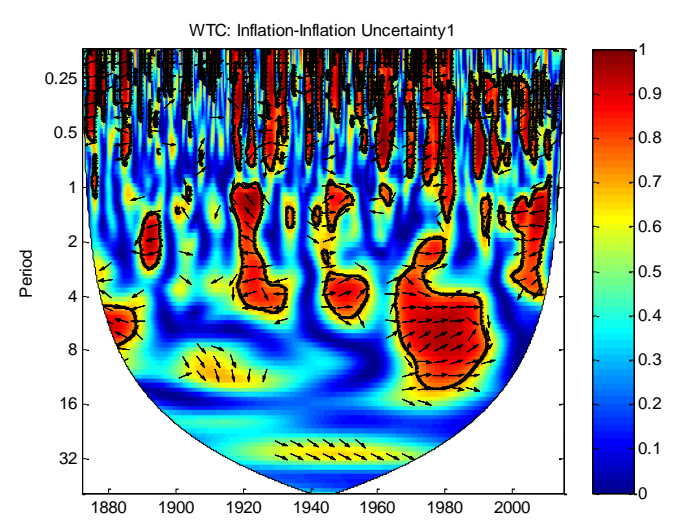

(b)

Notes: The black contour designates the 5\% significance level. Y-axis measures frequencies or scales, from the shortest scale (1-year cycle) to the longest scale (32-years cycle). X-axis represents the time period, covering the interval 1872:01 to 2015:05. The phase differences between the two series are indicated by arrows. Arrows pointing to the right mean that the variables are in-phase (they increase and decrease together), to the right and up mean that the inflation is leading, and to the right and down mean that the inflation is lagging. Arrows pointing to the left mean that the variables are out-of-phase, to the left and up mean that the inflation is lagging and to the left and down mean that the inflation is leading.

Figure 5. The XWT of the inflation series and its uncertainty (monthly data) 


\section{Appendixes}

\section{Appendix A - Wavelet analysis relying on the Stock and Watson's approach for}

\section{uncertainty (annual data)}
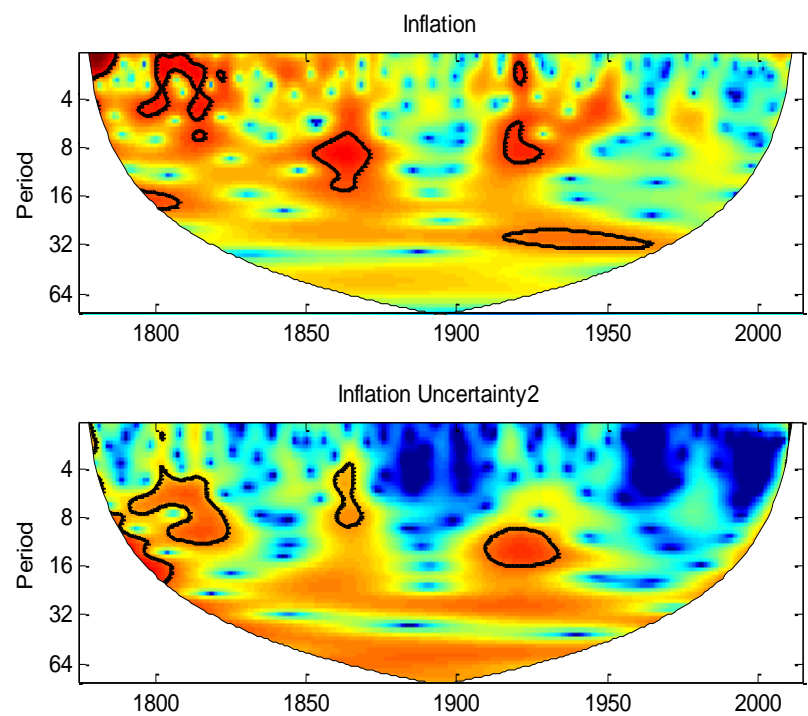

Notes: The black contour designates the 5\% significance level. Y-axis measures frequencies or scales, from the shortest scale (1-yearcycle) to the longest scale (64-years cycle). X-axis represents the time period, covering the interval 1775 to 2014.

Figure A1. The WPS of the inflation and uncertainty (Stock and Watson's approach, annual data)

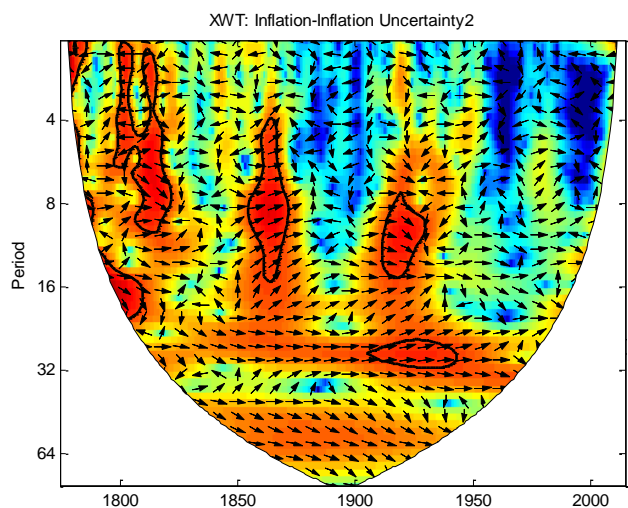

(a)

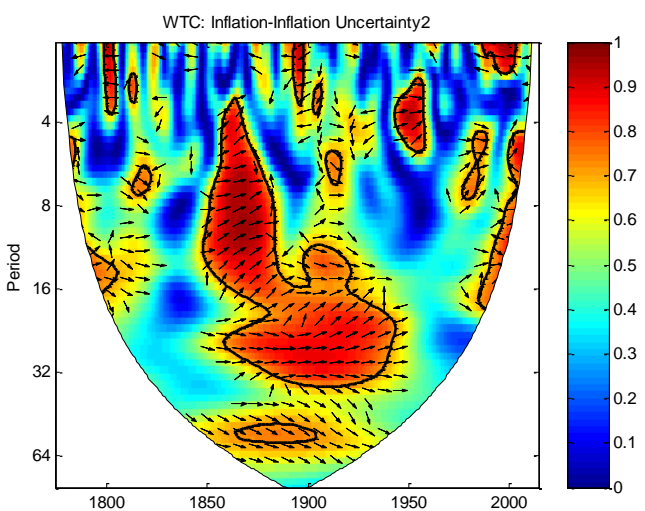

(b)

Notes: The black contour designates the 5\% significance level. $Y$-axis measures frequencies or scales, from the shortest scale (1-yearcycle) to the longest scale (64-yearscycle). X-axis represents the time period, covering the interval 1775 to 2014. The phase differences between the two series are indicated by arrows. Arrows pointing to the right mean that the variables are in-phase (they increase and decrease together), to the right and up mean that the inflation is leading, and to the right and down mean that the inflation is lagging. Arrows pointing to the left mean that the variables are out-of-phase, to the left and up mean that the inflation is lagging and to the left and down mean that the inflation is leading.

Figure A2. The XWT of the inflation and uncertainty

Stock and Watson's approach, annual data) 


\section{Appendix B - Wavelet analysis relying on the Stock and Watson's approach for uncertainty (monthly data)}
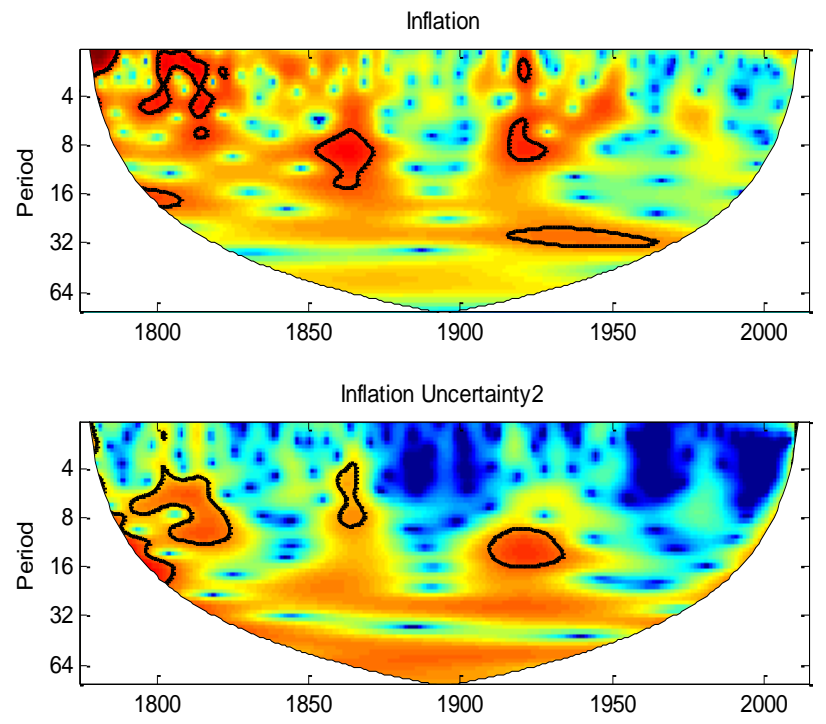

Notes: The black contour designates the 5\% significance level. Y-axis measures frequencies or scales, from the shortest scale (1-year cycle) to the longest scale (32-years cycle). $X$-axis represents the time period, covering the interval 1872:01 to 2015:05.

Figure B1. The WPS of the inflation and uncertainty (Stock and Watson's approach, monthly data)

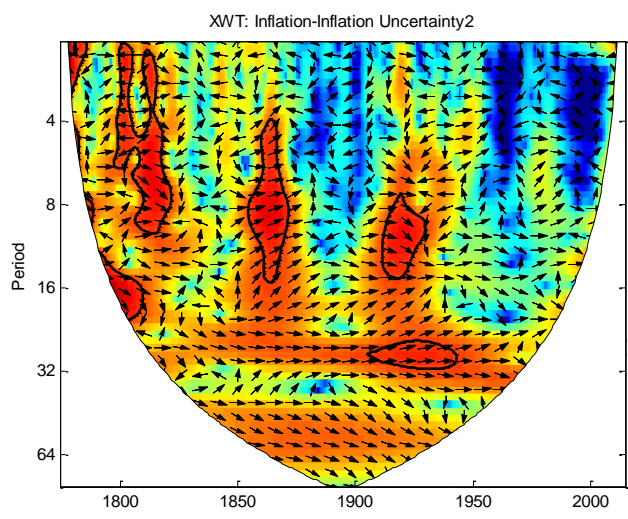

(a)

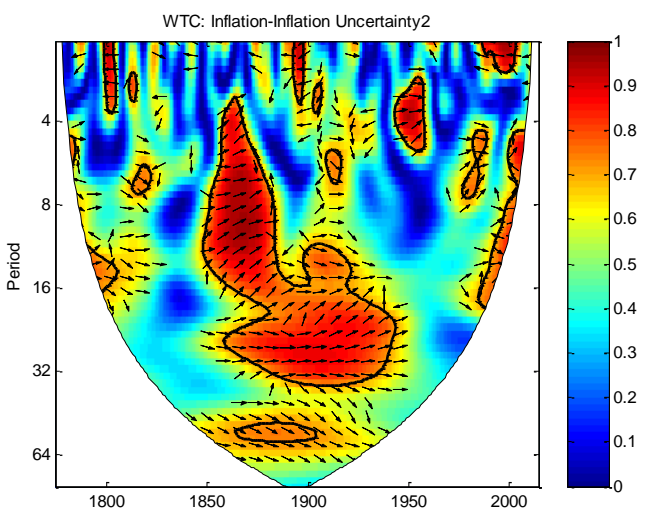

(b)

Notes: The black contour designates the 5\% significance level. $Y$-axis measures frequencies or scales, from the shortest scale (1-year cycle) to the longest scale (32-years cycle). X-axis represents the time period, covering the interval 1872:01 to 2015:05. The phase differences between the two series are indicated by arrows. Arrows pointing to the right mean that the variables are in-phase (they increase and decrease together), to the right and up mean that the inflation is leading, and to the right and down mean that the inflation is lagging. Arrows pointing to the left mean that the variables are out-of-phase, to the left and up mean that the inflation is lagging and to the left and down mean that the inflation is leading.

Figure B2. The XWT of the inflation and uncertainty

(Stock and Watson's approach, monthly data) 